\title{
ON THE STABILITY OF LINEAR SYSTEMS WITH DELAYED STATE DEFINED OVER FINITE TIME INTERVAL
}

\author{
D. Lj. Debeljković, S. A. Milinković, , M. B. Jovanović \\ University of Belgrade, System Control Group, Karnegijeva 4, 11000 Belgrade, Yugoslavia. \\ E-mail: emilinko@ubbg.etf.bg.ac.yu \\ Z. Lj. Nenadić \\ Washington University in St. Louis, St.Louis, MO 63112, USA.
}

\begin{abstract}
Paper extends some basic results from the area of finite time and practical stability to linear, continuous, time invariant time-delay systems. Sufficient conditions of this kind of stability, for particular class of time-delay systems are derived.

\section{Introduction}

Weiss and Infante $[1,2]$ have introduced various notations of stability over finite time interval for continuous-time systems and constant set trajectory bounds. Further development of these results were due to many other authors. In the context of finite time and practical stability, for the linear time delay system, various results were for the first time obtained by Nenadić et al. [3] and Debeljković et al. [4]. Further extensions of these results were due to Debeljkovic et al. [5] and Lazarevic et al. [6].
\end{abstract}

\section{Preliminaries}

A linear, multivariable time-delay system can be represented by differential equation:

$$
\dot{\mathbf{x}}(t)=A_{0} \mathbf{x}(t)+A_{1} \mathbf{x}(t-\tau),
$$

and with associated function of initial state:

$$
\mathbf{x}(t)=\Psi_{x}(t), \quad-\tau \leq t \leq 0,
$$

Equation (1) is referred to as homogenous or the unforced state equation, $\mathbf{x}(t)$ is the state vector, $A_{0}$ and $A_{1}$ are constant system matrices of appropriate dimensions, and $\tau$ is pure time delay, $\tau=$ const. $(\tau>0)$.

Dynamical behavior of autonomous system (1) is defined over time interval $J=\left\{t_{0}, t_{0}+T\right\}$, where quantity $T$ may be either a positive real number or symbol $+\infty$, so finite time stability and practical stability can be treated simultaneously. It is obvious that $J \in R$.

Let index $\beta$ stands for the set of all allowable states of system and index $\alpha$ for the set of all initial states of the system, such that the set $S_{\alpha} \subseteq S_{\beta}$. In general, one may write:

$$
S_{\rho}=\left\{x:\|x\|_{Q}^{2}<\rho\right\},
$$

where $Q$ will be assumed to be symmetric, positive-definite, real matrix.

Let $|\mathbf{x}|_{(.)}$be any vector norm (e.g., $\left.\cdot=1,2, \infty\right)$ and $\|(\cdot)\|$ the matrix norm induced by this vector. For our purpose, we use $|\mathbf{x}|_{2} \stackrel{\Delta}{=}\left(\mathbf{x}^{T} \mathbf{x}\right)^{1 / 2}$ and $\|(\cdot)\|_{2}=\lambda_{\max }^{1 / 2}\left(A^{*} A\right)$.

The matrix measure $\mu$ for any matrix $A \in \mathrm{C}^{n \times n}$ is defined as follows

$$
\mu(A) \stackrel{\Delta}{=} \lim _{\varepsilon \rightarrow 0} \frac{\|1+\varepsilon A\|-1}{\varepsilon} .
$$

For our purpose we use:

$$
\mu_{2}(A)=\frac{1}{2} \max _{i} \lambda_{i}\left(A^{*}+A\right),
$$

\section{Previous results}

Definition 1: System given by (1) satisfying initial condition (2) is finite time stable w.r.t. $[\zeta(t), \beta, T]$ if and only if:

implies:

$$
\psi_{x}^{T}(t) \psi_{x}(t)<\zeta(t)
$$

$$
\mathbf{x}(t)^{T} \mathbf{x}(t)<\beta
$$

$\zeta(t)$ being scalar function with the property $0<\zeta(t) \leq \alpha$, $-\tau \leq t \leq 0$, where $\alpha$ is a real positive number and $\beta \in R, \beta>\alpha$.

Theorem 1: The system given by (1) with the initial function (2) is finite time stable with respect to $\{\alpha, \beta, \tau, T\}$, if the following condition is satisfied:

$$
\|\Phi\|<\frac{\sqrt{\beta / \alpha}}{1+\tau\left\|A_{1}\right\|_{2}}, \quad \forall t \in[0, T]
$$

where $\|(\cdot)\|$ is Euclidean norm and $\Phi(t)$ is fundamental matrix of system (1), Nenadić et al. [3], Debeljković et al. [4].

Theorem 2. The system given by (1) with initial function (2) is finite time stable w.r.t. $\{\alpha, \beta, \tau, T\}$ if the following condition is satisfied

$$
e^{\mu_{2}\left(A_{1}\right) t}<\frac{\sqrt{\beta / \alpha}}{1+\tau\left\|A_{1}\right\|_{2}}, \quad \forall t \in[0, T]
$$


where $\|(\cdot)\|$ denotes Euclidean norm, Debeljković et al. [5]. For the sake of brevity proofs are here omitted.

\section{Main results}

Definition 2: System given by (1) satisfying initial condition (2) is finite time stable w.r.t. $[\zeta(t), \beta, T]$ if and only if:

$$
\left|\psi_{x}(t)\right|_{2}<\zeta(t)
$$

implies:

$$
|\mathbf{x}(t)|_{2}<\beta, \quad \forall t \in J
$$

$\zeta(t)$ being scalar function with the property $0<\zeta(t) \leq \alpha$, $-\tau \leq t \leq 0$, where $\alpha$ is a real positive number and $\beta \in R, \beta>\alpha$.

Theorem 3: The system given by (1) with the initial function (2) is finite time stable with respect to $\left\{\alpha, \beta, \tau, T, \mu_{2}\left(A_{0}\right) \neq 0\right\}$, if the following condition is satisfied:

$$
e^{\mu_{2}\left(A_{0}\right) t}<\frac{\beta / \alpha}{1+\mu_{2}^{-1}\left(A_{0}\right)\left\|A_{1}\right\|_{2}\left(1-e^{-\mu_{2}\left(A_{0}\right) r}\right)}, \forall t \in[0, T]
$$

where $\|(\cdot)\|_{2}$ denotes Euclidean norm.

Proof. The solution of (1) with initial function (2) can be expressed in terms of fundamental matrix as it is written below:

$$
\mathbf{x}(t)=\Phi(t) \psi_{x}(0)+\int_{-\tau}^{0} \Phi(t-\theta-\tau) A_{1} \psi_{x}(\theta) d \theta .
$$

Evaluating the norm $|(\cdot)|_{2}$ of both sides of this equations yields

$$
\begin{aligned}
|\mathbf{x}(t)|_{2} & \leq\|\Phi(t)\|_{2}\left|\psi_{x}(0)\right|_{2} \\
& +\left.\int_{-\tau}^{0}\|\Phi(t-\theta-\tau)\|_{2}\left\|A_{1}\right\||| \psi_{x}(\theta)\right|_{2} d \theta
\end{aligned}
$$

Using the known inequality,

$$
\|\exp (A t)\|_{2} \leq \exp \{\mu(A t)\}, \quad t \geq 0
$$

we have

$$
\begin{aligned}
|\mathbf{x}(t)|_{2} \leq & e^{\mu_{2}\left(A_{0}\right) t}\left|\psi_{x}(0)\right|_{2} \\
& +\int_{-\tau}^{0} e^{\mu_{2}\left(A_{0}\right)(t-\theta-\tau)}\left\|\left.A_{1}\left|\|_{2}\right| \psi_{x}(\theta)\right|_{2} d \theta,\right.
\end{aligned}
$$

or, using (10)

$$
|\mathbf{x}(t)|_{2} \leq \alpha e^{\mu_{2}\left(A_{1}\right) r}\left(1+\left\|A_{1}\right\|_{2} \int_{-\tau}^{0} e^{-\mu_{2}\left(A_{1}\right)(\theta+\tau)} d \theta\right),
$$

and finally:

$$
|x(t)|_{2} \leq \alpha e^{\mu_{2}\left(A_{0}\right) r}\left\{1+\mu_{2}^{-1}\left(A_{0}\right)\left\|A_{1}\right\|_{2}\left(1-e^{-\mu_{2}\left(A_{1}\right) \tau}\right)\right\} .
$$

Applying the basic conditions of Theorem, equation (12), on the preceding inequality, one can get

$$
|\mathbf{x}(t)|_{2}<\beta, \quad \forall t \in[0, T]
$$

what has to be proved. When $\tau=0$ or $\| A_{\mathrm{H}_{2}}=0$, the problem is reduced to the case of the ordinary linear, differential systems. In particular case, when $\mu_{2}\left(A_{0}\right)=0$, we can give the following result.

Theorem 4. The system given by (1) with initial function (2) is finite time stable w.r.t. $\left\{\alpha, \beta, \tau, T, \mu_{2}\left(A_{0}\right)=0\right\}$ if the following condition is satisfied

$$
1+\tau\left\|A_{1}\right\|_{2}<\beta / \alpha
$$

Proof. Follows directly from Theorem 3 when $\mu_{2}\left(A_{0}\right)=0$.

\section{Conclusion}

In this paper a new criterion for non-Lyapunov stability of particular linear time delay systems is derived. It is delaydependent criterion and is expressed by a simple inequalities. This result represents further generaliza-tion of known results derived earlier and provide condition that is more applicable than those given in current literature, derived by the same authors.

\section{References}

[1] Weiss, L., E. F. Infante (1965). "On the Stability of Systems Defined over Finite Time Interval", Proc. National Acad. Science, 54 (1) (1965) 44-48.

[2] Weiss, L., E. F. Infante (1967). "Finite Time Stability under Perturbing Forces on Product Spaces", IEEE Trans. on Automat. Cont., AC-12 (1) (1967) 54-59.

[3] Debeljković, D. Lj., Z. Lj. Nenadić, S. A. Milinković, "On Practical Stability of Time Delay Systems", Proc. ACC97, Albuquerque, New Mexico (USA), June 4-6 1997, pp. $3235-3236$.

[4] Nenadić, Z. Lj., D. Lj. Debeljković, S. A. Milinković, M. B. Jovanović, "On Practical and Finite-Time Stability of Time-Delay Systems", Proc. ECC97, Brussels (Belgium), July 1-4 1997, pp. 307-311.

[5] Debeljković, D. Lj., Z. Lj. Nenadić, Dj. Koruga, S. A. Milinković, M. B. Jovanović, "On Practical Stability of Time-Delay Systems: New Results", Proc. 2nd ASCC97, Seoul (Korea), July 22-25 1997, pp. III- 543-546.

[6] Lazarević, M. P., D. Lj. Debeljković, Z. Lj. Nenadić, S. A. Milinković, "Finite Time Stability of Time Delay Systems", IMA J. of Math. Control and Information, submitted (1997). 